\title{
La evolución del factor de impacto de Neurocirugía en su contexto nacional e internacional
}

\author{
J. Ibáñez
}

Servicio de Neurocirugía. Hospital Universitario Son Dureta. Palma de Mallorca.

La utilización de los indicadores bibliométricos para la evaluación de la calidad científica, y entre ellos del popular factor de impacto, debe realizarse con suma cautela. En su origen, el factor de impacto fue diseñado como un método de valoración de la repercusión de las revistas científicas dentro de su área específica de conocimiento. Las extrapolaciones tan habituales hoy en día hacia la evaluación individual de los trabajos, la importancia de sus autores, de sus departamentos o instituciones, o la comparación de publicaciones de distintas disciplinas deben realizarse con gran precaución cuando no ser directamente consideradas como metodológicamente incorrectas. Existe un sinfín de razones para denunciar la tiranía del factor de impacto y su incorrecto -pero frecuente empleo en tareas evaluadoras de la valía científica- pero no procede aquí detallarlas. Sin embargo, si hacemos un ejercicio de realismo, y nos movemos hacia posiciones pragmáticas, es un hecho incuestionable que el factor de impacto es hoy la divisa internacional con la que se valora y se efectúan las transacciones en este ámbito. Podrá gustarnos más o menos, podremos proponer ésta o aquella reforma en los métodos de evaluación, pero más nos vale conocer las reglas del juego actual para no desaparecer o quedar aislados en la dinámica vigente, donde este parámetro ha sido internacionalmente asumido desde hace ya tiempo, entre otras cosas, por la carencia de otros indicadores objetivos para desarrollar esta tarea.

Haciendo estas salvedades previas, el objetivo central de este breve informe es analizar el estado actual de la revista Neurocirugía desde este punto de vista, mostrando su situación actual, su evolución en los últimos años, así como las principales causas de ello. Aportaré también algunas reflexiones personales sobre estos datos, con la intención de ayudar a definir la mejor estrategia a seguir.

El primer análisis concierne a la posición de la revista dentro del contexto nacional de publicaciones evaluadas por el Institute for Scientific Information (ISI). Esta institución incluye un total de 29 revistas españolas en la edición de 2004. Neurocirugía ocupa el vigésimotercer puesto por factor de impacto $(0,299)$, el vigésimooctavo por número

Recibido: 10-04-06 total de citas recibidas (90) y el vigésimoprimero por número de artículos publicados (41). ¿Quiere ésto decir que estamos a la cola de la edición científica en español? Miremos los datos de otra forma. Preguntémonos cuántas, de las revistas españolas de especialidades quirúrgicas existentes, evalúa el ISI y veremos como la respuesta es muy indicativa: solamente una, la nuestra. Así pues, bajo esta perspectiva, todas las demás estarían por detrás, ya que ni siquiera aparecen indexadas. En segundo lugar, centrémonos en el campo de la neurología clínica y especialidades afines, área en la que deberíamos también situar nuestra publicación. Solamente cuatro revistas españolas podrían encuadrarse dentro de este epígrafe (Neurología, Revista de Neurología, Actas Españolas de Psiquiatría y Neurocirugía). Aquí Neurocirugía aparece como la segunda publicación por factor de impacto por delante de la todopoderosa "revista amarilla". ¿Significa esto ahora que nuestra revista no está tan mal? Cabría preguntarse a continuación, cómo es posible que una revista quincenal con una difusión tan amplia como la que tiene Revista de Neurología, que es órgano de expresión de 20 sociedades científicas españolas e iberoamericanas, y con la que es imposible que nosotros podamos competir desde el punto de vista económico, caiga casi un $30 \%$ por debajo de nuestra humilde publicación en términos de factor de impacto. Puede parecer que algo falla aquí, y que el factor de impacto no está evaluando bien a nuestros colegas. La razón para esta aparente contradicción debe buscarse en el modelo de publicación elegido, invitándonos a una reflexión sobre cual es el camino más adecuado a seguir en nuestro caso. El factor de impacto premia la publicación de artículos de calidad, entendiendo como tales aquéllos que contribuyen a la creación de nuevo conocimiento siendo citados por nuevos autores, y penaliza el exceso de información no relevante. Revista de Neurología publica mucho (realmente muchísimo). En concreto 859 artículos en los años 2002 y 2003 que fueron citados en 180 ocasiones durante 2004 dándole un factor de impacto de 0,210. Neurocirugía, por el contrario publica mucho menos, 97 trabajos en este periodo, habiendo recibido 29 citas, lo que supone un factor de impacto de 0,299. Las cifras hablan del peso y presencia de una y otra publicación 
$\mathrm{y}$, sin embargo, el premio en forma de factor de impacto que cosechan los autores que publican en cada una de ellas es, a día de hoy, sensiblemente favorable a nuestra revista. Personalmente creo no que el modelo elegido por Revista de Neurología, no debe ser el utilizado por nosotros. De forma sintética, con una oferta estable de trabajos, si hay que elegir entre publicar más o publicar menos, la segunda opción parece más razonable. Ser selectivos, publicando trabajos de mayor calidad cada vez, sin obsesionarnos con la cantidad, tiene que ser un objetivo prioritario de nuestra revista, y ello no está reñido con potenciar una difusión cada vez mayor de la misma. Para ello, la revista debe darse a conocer más, y ofrecerse como medio de comunicación a todos los neurocirujanos de habla española.

La evolución del factor de impacto de Neurocirugía en los últimos diez años es buena y refleja una clara progresión (Figura 1). Como ya he dicho, y aunque pueda sorprendernos, en el año 2004 el factor de impacto de Neurocirugía supera a otras revistas nacionales del área, situándose cada vez más cerca de otras publicaciones europeas del ámbito de la neurocirugía en habla no inglesa (Figura 2). Pero, ¿por qué ha aumentado el factor de impacto de Neurocirugía de esta forma tan llamativa? Mi opinión es que esto se debe fundamentalmente a dos razones: por un lado, la presencia en Medline ha aumentado de forma muy sensible las posibilidades de que los autores encuentren las referencias de los trabajos publicados por la revista, y la posibilidad de acceder de forma directa y gratuita a partir de la propia página de PubMed a estos artículos facilita la disponibilidad de los mismos; en segundo lugar, porque, al menos, un grupo de autores españoles ya está prestando atención a los trabajos publicados en la revista a la hora de fijar las citas de los nuevos artículos que elaboran. Ambos elementos son apreciables en el análisis de la procedencia de citas a la revista que muestra la persistencia de una elevada tasa de autocitación, pero también el incremento paralelo de las citas de otra procedencia (Figura 3). En mi opinión se debe insistir y favorecer ambas vías, manteniendo la gratuidad de la versión online, y continuando sin desmayo la tarea de concienciación y fomento del compromiso de los neurocirujanos españoles con su publicación científica.

Seguir por el camino que lleva al aumento, lento pero progresivo, del prestigio de una pequeña revista como la nuestra, precisa disponer de un buen número de originales, evitando comprometer la continuidad de la publicación si se sube el listón de la calidad. Ninguna publicación científica de prestigio acepta el 100\% de los originales que recibe, más bien al contrario. La tarea de los revisores es aquí fundamental. Siguiendo esta fórmula, quizás algún día lejano podamos llegar tener la revista que queremos.

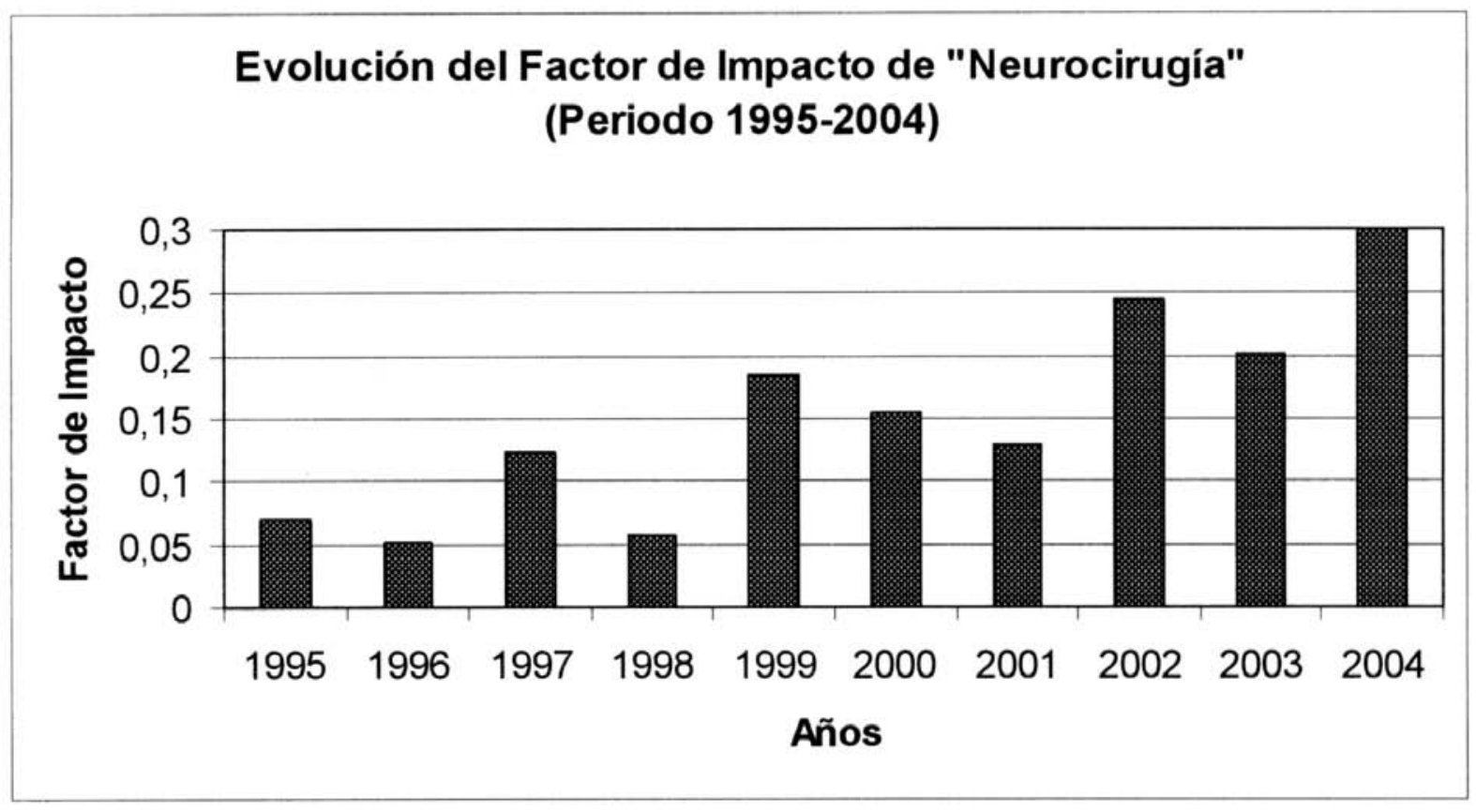

Figura 1. 


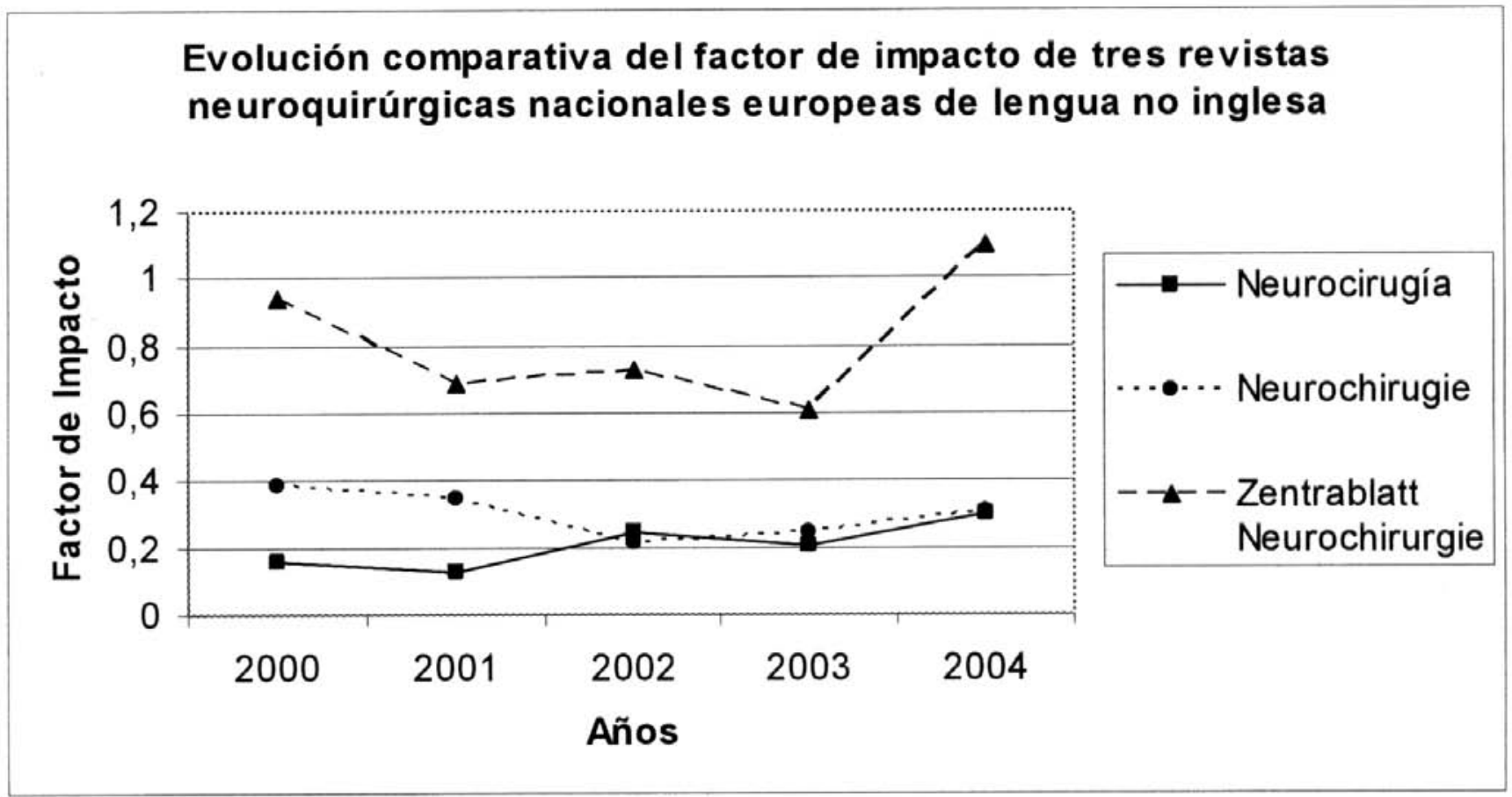

Figura 2.

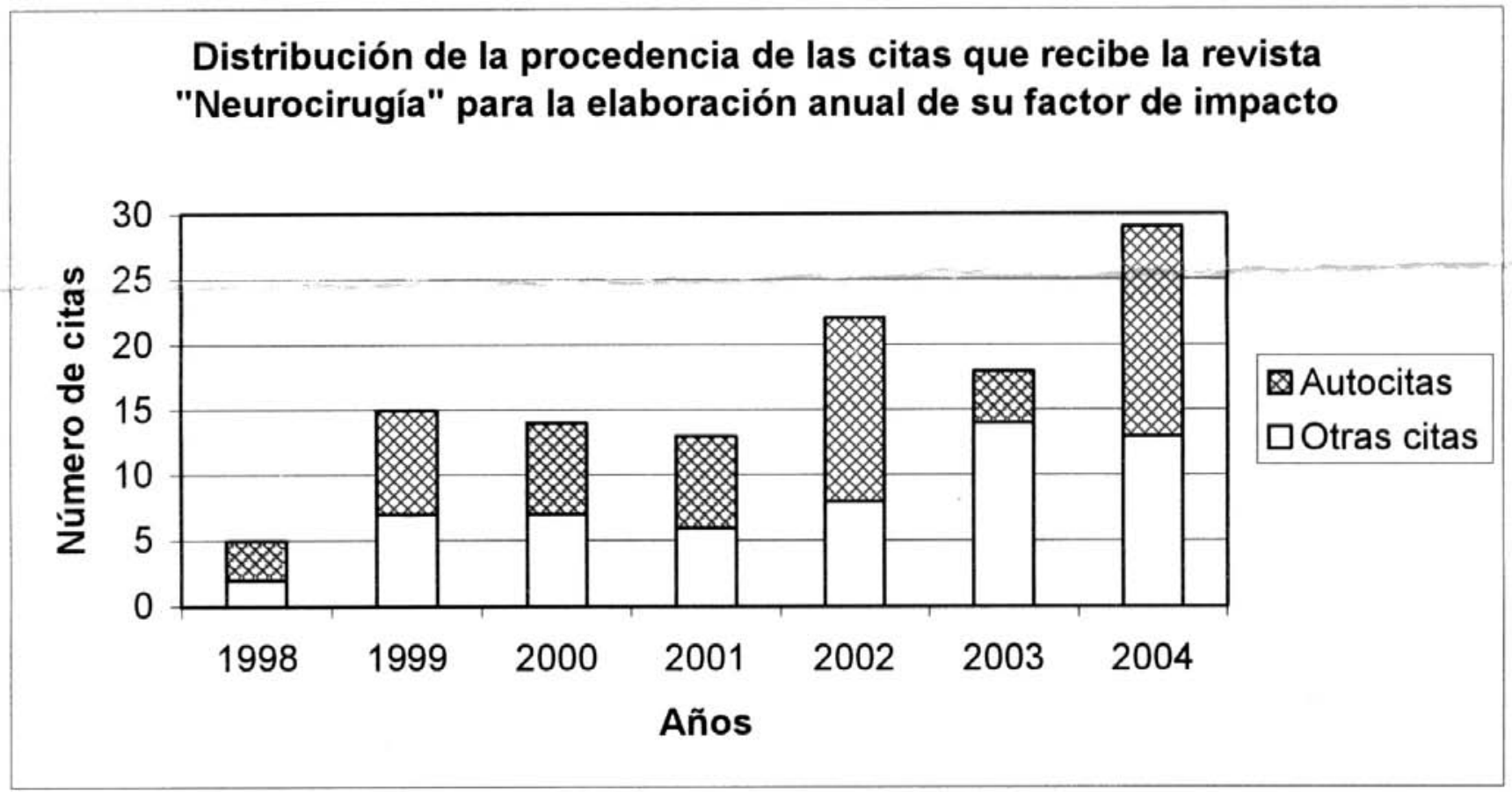

Figura 3. 
Otra cosa sería la receta para permanecer eternamente en la mediocridad. Aunque es natural aceptar que los autores que tienen en sus manos trabajos de alto nivel busquen su publicación en revistas de alto factor de impacto en sus áreas, ello no impide el llamamiento a la continuación y profundización de la atención de los neurocirujanos españoles hacia su revista nacional. Neurocirugía debe ser un instrumento fundamental en el progreso de la especialidad en nuestro país. A todos nosotros debería interesarnos cuidarla $\mathrm{y}$ hacerla crecer, con la seguridad de que Neurocirugía nos devolverá con creces el interés que en ella pongamos. Los potenciales autores de Neurocirugía deben saber que tienen a su disposición una revista que avanza, que les va a recompensar con un factor de impacto nada desdeñable, que está presente en Medline / Index Medicus, con vínculo directo y gratuito a la versión online de sus trabajos, ofreciéndoles así la máxima visibilidad y difusión. ¿Alguien puede pedir más para ponerse ya a escribir su próximo artículo?

J. Ibáñez. La evolución del factor de impacto de Neurocirugía en su contexto nacional e internacional. Neurocirugía 2006; 17: 98-101. 